\title{
Characteristics of Layered Gels Formation by Additive Technologies
}

\author{
Boris Pokusaev, Sergey Karlov, Andrey Vyazminª, Kseniya Okhotnikova and Dmitry Nekrasov \\ Moscow State University of Mechanical Engineering, Environmental and Chemical Engineering Institute, ul. Staraya \\ Basmannaya 21/4, Moscow, 105066 Russia
}

\begin{abstract}
In order to study the properties of the different density silica gels optical methods were used. It is shown that layers of gels spatially inhomogeneous and anisotropic, their optical density are significantly reduced near the free surface. It is found that on the surface of thin layers of silica gel there are convective structures. Features of multi-layered gel structures with different densities were studied. It is found that after the gels forming layers have poor adhesion to each other. This is due to the fact that the volume between different layers of gels filled with liquid. The presence of liquid in the interlayer volume is observed visually and confirmed by the light scattering of the laser beam. The thicknesses of the liquid boundaries between the layers of the gels were estimated.
\end{abstract}

\section{Introduction}

Gel is a dispersive system with liquid dispersing medium, and the dispersion phase makes up a spatial structured mesh due to intermolecular interaction in the contact sites. Gels are micro- or nanostructured media whose internal structure depends on the material and on the method of preparation. The characteristics of gel can change due to the internal processes $[1,2]$.

The promising application of gels is the use in regenerative medicine for growth of biological tissues, including the problem of culturing stem cells in vitro [3, 4]. This capillary network is intended for the delivery of nutrients to individual cells, and to remove products from the metabolism. It seems promising the idea that gels can used as a carrier during the formation of ordered bio-structured elements (based on the 3D printing) for additive medical technology. However, there are a number of factors that influence diffusion transport in gels and change mass-transfer laws [5, 6].

A large number of studies concerning the synthesis of different chemical nature gels and the study of its properties have been published. However, for use in technology gels have many common properties. For example, the most important properties that define the processes of mass transfer in the gels are nonstationarity and anisotropy due to the structure and behavior of the transfer medium.

For experimental study we used gels that are based on silicates. Such gels are well investigated (see, for example [7]), simply prepared and not toxic. Such gels are appropriate for additive technologies used for the creation of skeleton matrix (retaining biologically active gels, but transmissive to liquid nutrient) of bioreactors by 3D-printing. Gels of different density were investigated. The stock solution of sodium silicate was diluted with distilled water to the levels of density $1.04-1.08 \mathrm{~g} / \mathrm{cm}^{3}$. The gel-generating mixture was prepared by adding the hydrochloric acid.

\footnotetext{
${ }^{a}$ Corresponding author: av1958@list.ru
} 
The aim of the investigation is to study of the fundamental properties of the gels layers by optical methods (for example, silica gels different density) in relation to its using in additive 3D technologies. It is important to obtain data on the properties of multilayer gels structures and to determine the effects on the properties of the boundary layers between the gels.

\section{Nonuniformity and anisotropy of layered gel}

For the investigation of the anisotropic properties of gel the spectroscopic method was used. A container for a gel has produced from special quartz glass. The experimental setup provides the instantaneous registration in spectral range of $200-1100 \mathrm{~nm}$. The optical resolution is in average 1.0 $\mathrm{nm}$. The spatial resolution is $1 \mathrm{~mm}$. This setup allows us to investigate the intensity of the transmitted light through the gel as a function of the distance from the top surface. Thickness of wetting meniscus is about $1.5 \mathrm{~mm}$. For this reason, measurements were made starting at a depth of $2 \mathrm{~mm}$.

The intensity of the light passing through the gel, referred to the value corresponding to the transmittance intensity of the optical system without gel. Measurement processed for light wavelength of $500 \mathrm{~nm}$, that corresponds to the minimum transmittance of the gels. The results of the measurements for gels with two different initial densities are shown in Fig. 1.

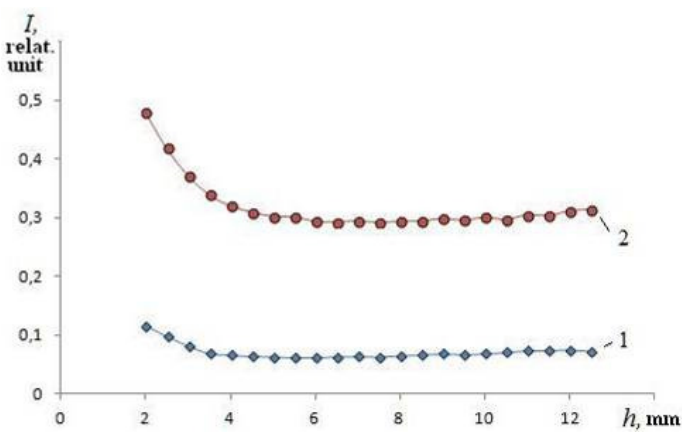

Figure 1. Intensities of transmitted light $I$ (relat. units) vs. distance from the top surface of the gel layer $h(\mathrm{~mm})$ for different density: $1-1.08 \mathrm{~g} / \mathrm{cm}^{3} ; 2-1.04 \mathrm{~g} / \mathrm{cm}^{3}$. Measurements were carried out 1 hour after gel formation.

From the experimental data it can be concluded that gels of both densities are nonuniform in their thickness. The density of the surface layers of the gel can be less than their density inside the gel. The explanation of this fact, that describes the temporal dynamics of the process of syneresis, is the slow deposition of a microstructured phase in a liquid under the forces of gravity. The deposition rate is determined by the rate of filtration of the liquid phase from the lower layers of gel to the top.

The formation of silica gel from liquid media requires a few minutes and it is associated with chemical changes. As a result a structured phase is occurred; gel has different properties from original liquids. It is found that on the surface of thin layers of silica gel there are convective structures (Fig. 2). These structures make more complicated to depose the upper layers of the gel at bioprinting.

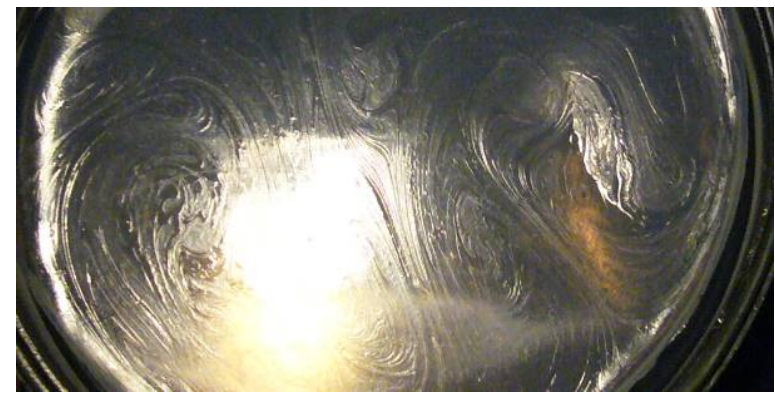

Figure 2. Photo of the convective structures appearing on the surface of a thin layer of silica gel during its formation. 


\section{Specific properties of multilayer gels}

In order to create 3D additive technologies using gels, there is a need to check whether the properties of additivity are valid for multilayered gels. For this purpose it is required to determine which properties of multilayer gels differ from the homogeneous gel of the same chemical composition.

Gels are very promising for additive technologies, since they are easily form matrixes of different shapes and densities (Fig. 3). It is possible to apply a denser layer of gel on the less dense without any mixing corresponding to liquid media. Such bilayer systems are hydrodynamic stable. For layered gel structures presented on pictures the border between the layers (pointed by arrow) is clearly visible.

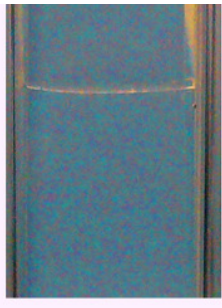

a



b


$\mathrm{C}$

Figure 3. Photos of silica gel two-layer systems of different densities: a - both layers with density of $1.04 \mathrm{~g} / \mathrm{cm}^{3}$, $\mathrm{b}$ - both layers with $1.08 \mathrm{~g} / \mathrm{cm}^{3}, \mathrm{c}$ - the upper layer has the density of $1.08 \mathrm{~g} / \mathrm{cm}^{3}$; the lower has the density 1.04 $\mathrm{g} / \mathrm{cm}^{3}$. The arrow indicated the separation surface between these two layers.

A comparison of the spectrum of single-layered and double-layered gel structures of the same thickness did not show differences in the intensity of transmitted light, either in the form of spectrum. However, the presence of interphase boundaries may have an impact on the technological properties of gels. In Fig. 4 shows the mechanical properties of the gels after drying. On the left there is the initial lattice structure created from the fully formed gel, on the right - inadvertent mechanical disruption of this structure after the day as a result of the drying of the gel. Breaks the continuity of the patterns observed in the contact layers. This indicates poor adhesion between the layers of the gel.
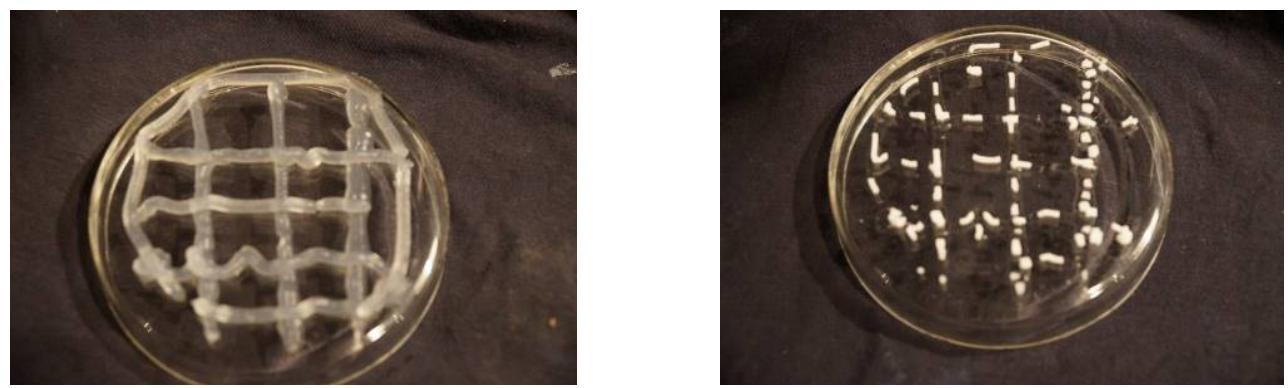

Figure 4. Photo of gel grating (left) on Petri dish, the same grating after drying of gel for 24 hours (right).

Important factor complicating the use of additive technologies to gels is their spontaneous densification. As a result of the pressure of the upper layers of the liquid is squeezed out of the lower layers forming the system drops on the perimeter of the upper layer (Fig. 5). Such a drop will obstruct the uniform application of the subsequent layers of the gel.

To make sure that between layers of the gel there is a thin layer of fluid, a study of impact of passing the green laser with wavelength $532 \mathrm{~nm}$ through it was performed. The main results are shown in Fig. 6. The passage of the beam through a homogeneous gel is uniform in all directions scattering of light, which fully corresponds to the scattering of light on the microinhomogeneities smaller wavelengths of light. When the ray go along the boundary layers of the gels it is clearly visible a lateral dispersion that occurs in a planar waveguide. This phenomenon is caused by the multiple reflections of light on the surfaces of the gels formed, that are limited by layer of liquid. 


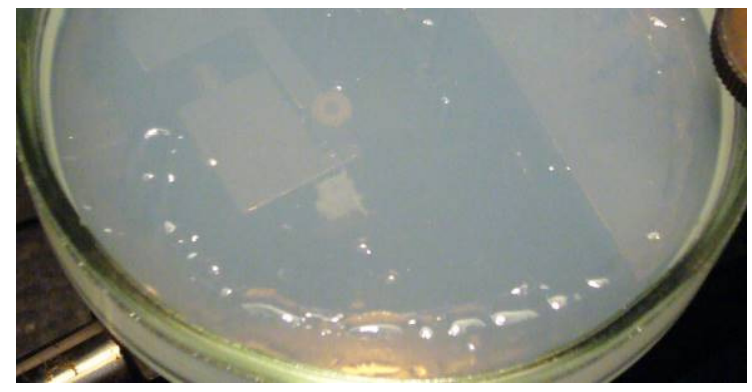

Figure 5. Photo of drops formed along the perimeter of upper layer during the formation gel two-layer matrix.

In order to estimate the thickness of the liquid layer between the gels a photographic method was used. Since the space between layers of the gel is a thin capillary, for receiving the contrast photos the inert colored dye was used that is well identified by spectral processing photo. The estimation showed that the thickness of the layer between gels is at least $0.5 \mathrm{~mm}$ and increases with aging of the gel.
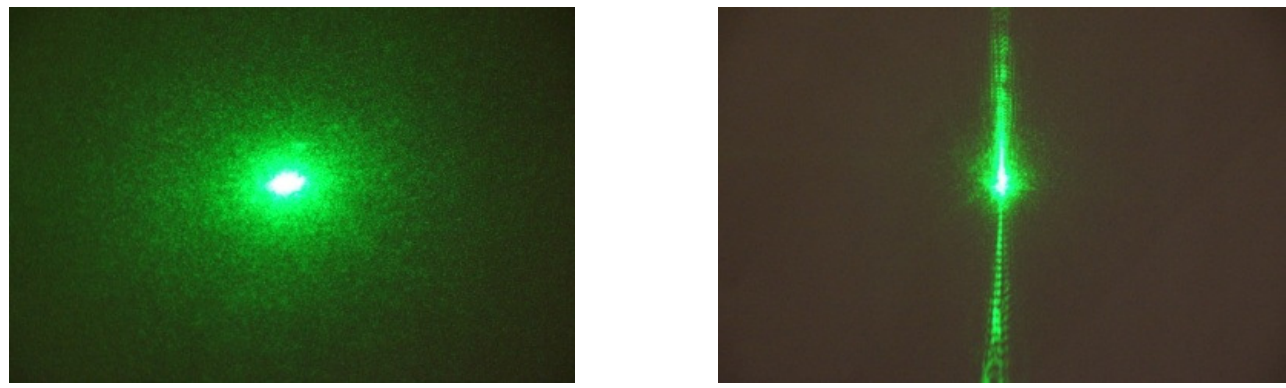

Figure 6. Photo of characteristic scattering of laser beam of $532 \mathrm{~nm}$ passing through a homogeneous gel (left), the characteristic scattering of the laser beam passing through the boundary between the gels layers (right).

\section{Conclusions}

Layers of gels are not uniform in thickness. The density of gels decreases towards the free surface. This is true for gels of different density. On the free surface of the gel during its formation, the occurrence of spontaneous interfacial convection is possible.

Multilayered gels are different in properties from a single layered of the same thickness. Between the layers of gel a thin fluid-filled layer is formed. This layer reduces adhesion between the layers of gel, but can significantly increase mass transfer of gel matrix. These features of layered gel structures must be taken into account when using such materials in additive $3 \mathrm{D}$ printing technology.

\section{Acknowledgement}

This work was supported by the Russian Science Foundation (project no. 15-19-00177).

\section{References}

1. K. Kajiwara, Yo. Osada, Gels handbook (Elsevier, 2000)

2. M. Tokita, K. Nishinary, Prog. Coll. \& Polymer Sci. 136, (2009)

3. B. A. Westrin, A. Axelsson, Biotech. \& Bioeng. 38, 439 (1991).

4. J. A. Hendriks, L. Moroni, J. Riesle, J. R. De Wijn, C. A. Van Blitterswijk, Biomater. 34, (2013)

5. B. Pokusaev, S. Karlov, A. Vyazmin, D. Nekrasov, Chem. Eng. Trans. 43, 1681 (2015)

6. M. Lauffer, Biophys. J. 1, 205 (1961)

7. N. A. Shabanova, P. D. Sarkisov, Sol-gel technology. (Binom. Moscow, Russia, 2012) 